\title{
The prevalence of biofilms in chronic wounds: a systematic review and meta- analysis of published data
}

\section{Authors: Matthew Malone, Thomas Bjarnsholt, Andrew J. McBain, Garth A. James, Paul Stoodley, David Leaper, Masahiro Tachi, Gregory Schultz, Terry Swanson, \& Randall D. Wolcott.}

This is a postprint of an article that originally appeared in Journal of Wound Care on January 2017. DOI:10.12968/jowc.2017.26.1.20

Malone M, Barjnsholt T, McBain AJ, James GA, Stoodley P, Leaper D, Tachi M, Shultz G, Swanson T, Wolcott RD "The prevalence of biofilms in chronic wounds: A systematic review and meta-analysis of published data” Journal of Wound Care, 2017 Jan 2;26(1):20-25. 


\title{
The prevalence of biofilms in chronic wounds: a systematic review and meta-analysis of published data
}

\begin{abstract}
The presence of biofilms in chronic non-healing wounds, have been identified through in vitro model and in vivo animal data. However, human chronic wound studies are under-represented and generally report low sample sizes. For this reason we sought to ascertain the prevalence of biofilms in human chronic wounds by undertaking a systematic review and meta-analysis. Our initial search identified 554 studies from the literature databases (Cochrane Library, Embase, Medline). After removal of duplicates, and those not meeting the requirements of inclusion, nine studies involving 185 chronic wounds met the inclusion criteria. Prevalence of biofilms in chronic wounds was 78.2 \% (confidence interval [CI 61.6-89, $\mathrm{p}<0.002])$. The results of our meta-analysis support our clinical assumptions that biofilms are ubiquitous in human chronic non-healing wounds.

Declaration of interest: This work was supported with funding from Smith and Nephew. Authors received travel and accommodation expenses, together with honoraria for teaching and participation in advisory/consultation groups from Smith \& Nephew.
\end{abstract}

Keywords: biofilm • systematic review • wound healing • infection • non-healing

Unlike planktonic microorganisms, biofilm phenotypes have been defined as structured consortiums of aggregated microbial cells, surrounded by a polymer matrix, that adhere to natural surfaces, to artificial surfaces or to themselves. ${ }^{1}$ The concept of biofilms in human health and disease is now universally accepted in tuberculosis, ${ }^{2}$ periodontal disease and dental caries, ${ }^{3}$ cystic fibrosis, ${ }^{4-6}$ in-dwelling medical device infections, ${ }^{7}$ otitis media and other upper respiratory infections, 8,9 and chronic wounds. ${ }^{10,11}$ So highly attuned are researchers to the wide involvement of biofilm-associated infections across the spectrum of human health and disease, that the US Department of Defense, has recognised the significance of biofilm as being problematic in wound healing, and has prioritised research in this area. 12

Biofilm-phenotype microorganisms residing within a chronic non-healing wound may promote a hyper-inflammatory response, detrimental to the host. ${ }^{13-15}$ Recent observations using oxygen microsensors and transcriptomics (examining oxygen depletion in micro niches and microbial metabolic activities) have provided alternate insights suggesting that bacterial biofilm in chronic wounds may promote localised tissue hypoxia reducing the availability of oxygen required for wound healing. ${ }^{16}$

Once established, biofilm highly tolerant to standard removal/eradication paradigms mechanical debridement. ${ }^{17}$ often become

treatment and such as
Several features that distinguish biofilm phenotypes from their planktonic counterparts are their tolerance to antimicrobial agents, ${ }^{18}$ disinfectants and the host's immune defences. ${ }^{19,20}$

While non-healing chronic wounds represent an umbrella terminology for a range of pathologies, biofilm have been cited across all related aetiologies including venous leg ulcers (VLUs), ${ }^{21}$ pressure ulcers (PUs) ${ }^{14,22}$ and diabetic foot ulcers (DFUs). ${ }^{10}$ Collectively these chronic wounds contribute to significant morbidity, mortality and increased health-care expenditure. Importantly, the continuing rise in antimicrobial resistance has placed a greater emphasis on correctly diagnosing and managing biofilm-associated infections in non-healing chronic wounds. This will require a shift in treatment paradigms to more multifaceted biofilm-based approaches given the resilience of biofilms in responding to planktonic- 
Fig 1. PRISMA flow diagram of literature search

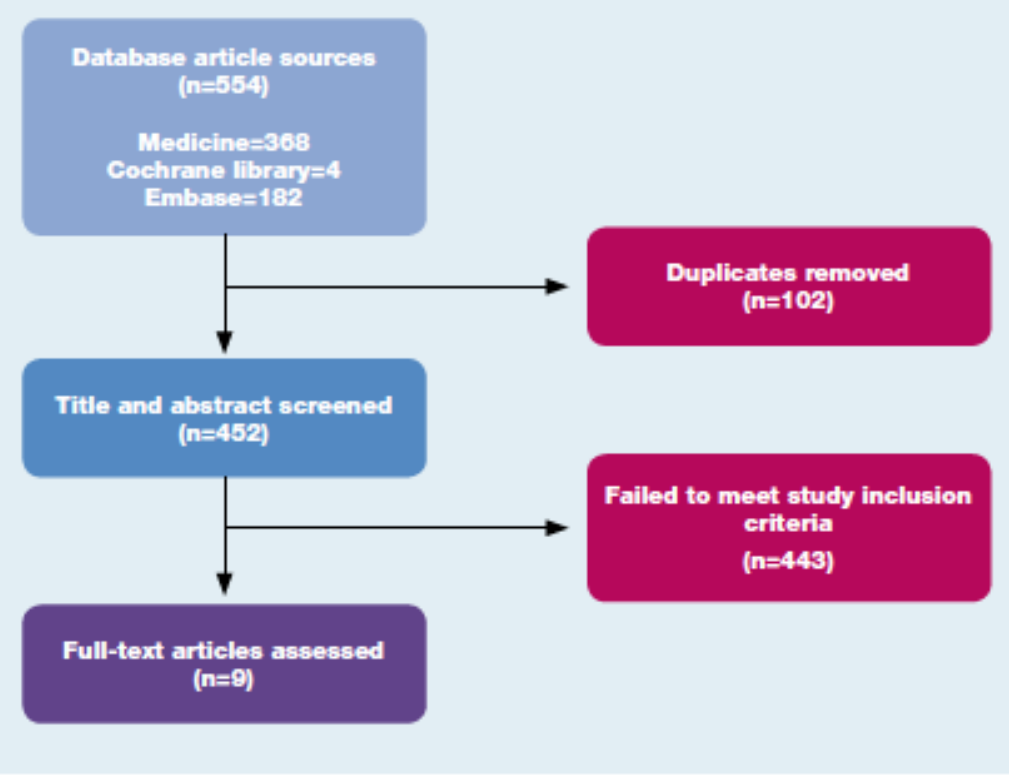

based treatments.

As the presence of biofilms across the spectrum of chronic wounds has significant implications both medically and economically, clear and concise information is required to help guide health-care professionals managing these recalcitrant causes of delayed healing. An increasing body of evidence from in vitro models and animal ${ }^{23,24}$ and human studies ${ }^{25}$ has identified the capacity of wound isolates to grow as biofilms, and for chronic non-healing wound samples to harbour biofilm. ${ }^{26}$ This has been driven largely by advancements in molecular microbiology, microscopy technology and techniques for the study of bacterial populations in situ. This has allowed authors to identify biofilm as the cause of non-healing chronic wounds and in the development of associated clinical infections.

The bulk of evidence supporting the notion that biofilm complicate non-healing chronic wounds is from in vitro model and in vivo animal data. ${ }^{27-30} \mathrm{~A}$ recent review of the scientific literature for the presence of biofilm in chronic wounds has eloquently explored the models used. ${ }^{31}$ However, human chronic wound studies are under-represented with most having low sample sizes. For this reason we aim to determine the prevalence of biofilm recognised in human chronic wounds by systematically reviewing the literature published on in vivo human chronic wound studies and to increase sample size and power by performing a meta-analysis.

\section{Methods}

\section{Search strategy}

A start date of 2008 was used based on a decision by the authors that this best represented the start of publications that used acceptable terminology and visualisation methods that best described and depicted microbial aggregates, extracellular polymeric substance (EPS) and the spatial orientation of microorganisms in samples. A systematic review of the Cochrane Library, Embase, and Medline (PubMed) databases was conducted for articles published between January 2008 and December 2015 using the following search terms: 'biofilm' [all fields] AND 'chronic wounds'.

A secondary search was undertaken using 'biofilm' with supplementary keyword filters: OR 'diabetic foot ulcers' OR 'venous leg ulcers' OR 'pressure ulcers' OR 'decubitus ulcers' OR 'non-healing surgical wounds', OR 'visualization', OR 'scanning electron microscopy' OR 'fluorescent in-situ hybridization', OR '16S rRNA'. Only articles in the English language were included. The search was limited to prospective clinical studies, case reports, case series and published conference abstracts. The systematic review was conducted in accordance with the PRISMA guidelines. ${ }^{32}$

\section{Data extraction}

Investigators (MM and TB) independently reviewed titles and abstracts of all articles to establish their eligibility on the basis of predefined criteria. All eligible article references were tabled and their abstracts obtained for review. Articles meeting the eligibility criteria were handsearched for additional studies. For the purpose of the meta-analysis, we extracted the following domains or variables from the articles: date of study publication (2008-2015), prevalence rates (number of confirmed tissue samples over the total number of samples screened), sample size and study design.

\section{Study eligibility}

Articles publishing data on in vivo human chronic wounds, in participants over the age of 18 , were included. Chronic wound aetiologies included in the search were DFUs, VLUs, PUs and non-healing surgical wounds (NHSWs). Individual searches of the methodology section were undertaken and universal definitions of a chronic wound or phrases denoting the chronicity of participant wounds such as 'non-healing', 'delayed healing' and/or 'chronic' were used to ensure eligibility.

Only articles detailing the presence of biofilm and bacteria in general through microscopy with or without combined molecular methods were included for review. In line with recent guidelines ${ }^{33}$ the following visualisation techniques were deemed appropriate for the confirmation of biofilm presence: scanning electron microscopy (SEM), transmission electron microscopy (TEM), confocal laser scanning microscopy (CLSM), conventional and peptide nucleic acid-fluorescent in situ hybridisation (PNA-FISH) and microscopy with or without staining methods. Articles diagnosing biofilm presence by clinical observation were excluded. Confirmation of biofilm included all visualisations of aggregated bacteria within the wound bed. ${ }^{34}$

Additionally, to meet inclusion criteria, articles must have cited optimal collection methods for the sampling of chronic wounds with tissue biopsy, 
Table 2. Random-effects model of nine chronic wound biofilm studies

\begin{tabular}{|c|c|c|c|c|c|c|}
\hline Author/s (year) & Study design & $\begin{array}{l}\text { Number of } \\
\text { participants }\end{array}$ & $\begin{array}{l}\text { Visualisation } \\
\text { methods with or } \\
\text { without molecular }\end{array}$ & $\begin{array}{l}\text { Chronic wound } \\
\text { aetiologies }\end{array}$ & $\begin{array}{l}\text { Numb } \\
\text { with c } \\
\text { biofiln }\end{array}$ & $\begin{array}{l}\text { of samples } \\
\text { firmed } \\
\% \text { ) }\end{array}$ \\
\hline James et al. $(2008)^{10}$ & $\begin{array}{l}\text { Prospective study } \\
\text { case versus control }\end{array}$ & 66 & $\begin{array}{l}\text { Light microscopy, } \\
\text { SEM 16S rRNA with } \\
\text { DGGE }\end{array}$ & $\begin{array}{l}13 \text { DFUs, } 21 \text { PUs } \\
8 \text { VLUs, } 24 \text { NHSWs }\end{array}$ & $30 / 50$ & $(60 \%)$ \\
\hline Kirketerp-Moller et al. $(2008)^{30}$ & $\begin{array}{l}\text { Prospective cohort } \\
\text { study }\end{array}$ & 22 & PNA-FISH, CLSM & Unspecified chronic wounds & $13 / 22$ & $(60 \%)$ \\
\hline Fazli et al. (2009) ${ }^{41}$ & $\begin{array}{l}\text { Prospective cohort } \\
\text { study }\end{array}$ & 9 & PNA-FISH, CLSM & 10 VLUs & $10 / 10$ & $(100 \%)$ \\
\hline Thomsen et al. $(2009)^{44}$ & $\begin{array}{l}\text { Prospective cohort } \\
\text { study, sub analysis }\end{array}$ & 2 & $\begin{array}{l}\text { PNA-FISH, } 16 \mathrm{~S} \\
\text { rRNA }\end{array}$ & 2 VLUs & $2 / 2$ & (100\%) \\
\hline Han et al. $(2011)^{38}$ & $\begin{array}{l}\text { Prospective cohort } \\
\text { study }\end{array}$ & 15 & $\begin{array}{l}\text { PNA-FISH, CLSM } \\
16 S \text { rRNA }\end{array}$ & $\begin{array}{l}4 \text { DFUs, } 5 \text { PUs, } 2 \text { VLUs } \\
4 \text { NHSWs }\end{array}$ & $9 / 15$ & $(60 \%)$ \\
\hline Neut et al. $(2011)^{43}$ & Case report & 2 & CLSM & 2 DFUs & $2 / 2$ & $(100 \%)$ \\
\hline Oates et al. $(2014)^{40}$ & $\begin{array}{l}\text { Prospective cohort } \\
\text { study, sub analysis }\end{array}$ & 4 & FISH, SEM & 4 DFUs & $4 / 4$ & $(100 \%)$ \\
\hline Martinez-Velasco et al. $(2014)^{36}$ & $\begin{array}{l}\text { Prospective cohort } \\
\text { study, conference } \\
\text { abstract }\end{array}$ & 20 & SEM, LM & Unspecified chronic wounds & $20 / 20$ & $(100 \%)$ \\
\hline Honorato-Sampaio et al. (2014) & $\begin{array}{l}\text { Prospective cohort } \\
\text { study }\end{array}$ & 45 & TEM & 45 VLUs & $45 / 45$ & $(100 \%)$ \\
\hline
\end{tabular}

curettage or debridement material being regarded as gold standard. Swab cultures of the wound bed were excluded for being inadequate for biofilm identification, given the inability to distinguish between planktonic and biofilm phenotype. ${ }^{33}$

\section{Statistical analysis}

Data from studies were extracted as raw numbers using the number of samples with confirmed biofilm over the total number of samples obtained. Data were analysed using comprehensive meta-analysis software (Biostat Inc., NJ, US). Prevalence estimate rates, weighted averages and $95 \%$ confidence intervals (CIs) were undertaken using fixed-effects meta-analysis. Forest plots were reported for inconsistencies in effect sizes and their CIs. Between-study variance or heterogeneity in estimates was modelled using Cochran's $Q$ and the $\mathrm{I}^{2}$ statistic. Where Cochran's Q value was reported with p-values less than 0.10 and $\mathrm{I}^{2}$ values exceeded $50 \%$, a random-effects model was used. ${ }^{35}$

\section{Results}

\section{Search results}

The search identified 554 studies from the literature databases. After removal of duplicates, exclusion and the screening of 452 titles and abstracts, eight studies involving 185 chronic wounds met the inclusion criteria (Fig 1). The numbers of each respective chronic wound were: DFUs $(n=33)$, VLUs $(n=67)$, PUs $(n=26)$,
NHSWs ( $n=28$ ), unspecified chronic wounds ( $n=31$ ). There were eight articles from prospective cohort studies with the remaining one study being case reports/series (Fig 2). Primary authors were contacted for data from two studies in order to clarify the number of positive biofilm samples. ${ }^{36,37}$ Between-study results identified heterogeneity ( $\mathrm{Q}$ test $\mathrm{p}<0.022, \mathrm{I}^{2}=55 \%$ ), to address this a random-effects model was used with prevalence rates reported.

\section{Prevalence of biofilms in chronic wounds}

The prevalence of biofilms in chronic wounds was $78.2 \%$ [CI 61.6-89, p<0.002]. Biofilm prevalence across studies identified the percentage(s) of positive biofilm samples was no lower than $60 \%$ (noted in three studies), ${ }^{10,38,39}$ with all remaining studies identifying $100 \%$ biofilm prevalence. ${ }^{36,37,40-43}$ Given the relatively small sample size and the covariable of four different chronic wound aetiologies, inferences regarding whether biofilms were more prevalent in one particular chronic wound were not possible.

\section{Discussion}

Early publications providing evidence for the presence of biofilm in chronic wounds have provided guidance for clinicians and researchers alike. ${ }^{10,11,39}$ These studies identified that biofilm were present in $60 \%$ of nonhealing chronic wounds. Since then, studies employing combined molecular and microscopy methods to directly 
Table 2. Random-effects model of nine chronic wound biofilm studies

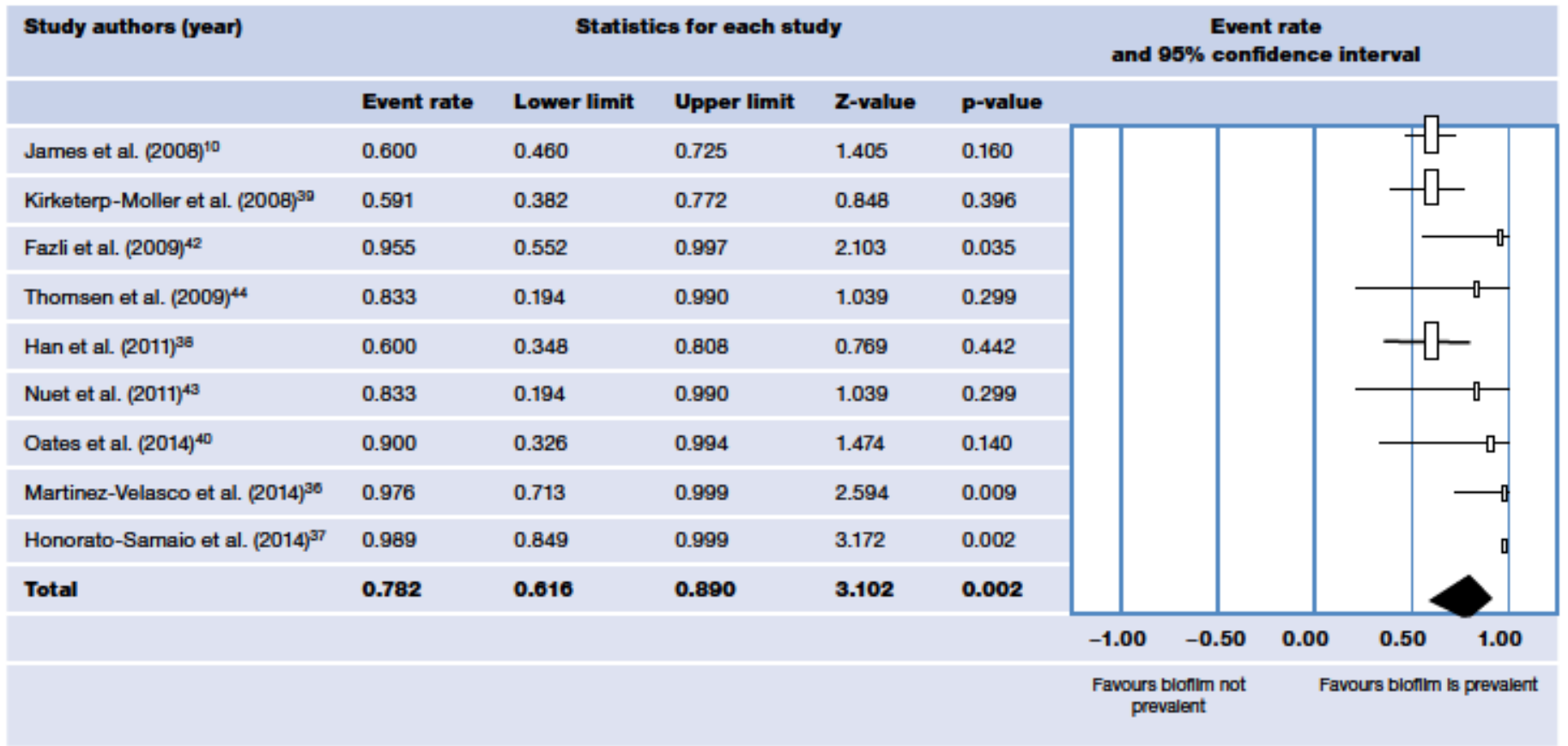

visualise biofilms have gathered pace.

This systematic review and meta-analysis has collated all available in vivo studies pertaining to the identification of biofilms from non-healing human chronic wounds. In doing so, our meta-analysis results suggest that biofilms are prevalent in all these wounds. Prevalence rates identify that $78 \%$ of non-healing chronic wounds harbour biofilms, with prevalence rates varying between $60 \%$ and $100 \%$. We propose, therefore, that biofilm are ubiquitous in nearly all nonhealing chronic wounds and the disparity in prevalence rates may be a reflection of study design and methodological limitations. For example, we argue that heterogeneous distribution of microorganisms within wounds may allow for variability in sampling, increasing the likelihood of returning negative or inconclusive samples.

Previous studies, ${ }^{39,44,45}$ have highlighted the heterogeneous spatial distribution of wound microbiota through sampling multiple areas of the wound bed, identifying vast shifts in community diversity. This suggests relying on a single site for sampling may reduce the chances of visualising biofilm. Obtaining samples from multiple sites of the wound may improve the detection of biofilm. However, this is often not feasible at a clinical level and is reflected in many studies that employ tissue collection methods.

The primary aim of this systematic review and meta-analysis was to provide a statistical approach for further justifying the evidence that biofilm are present in chronic non-healing wounds. We acknowledge that our analysis has obvious limitations, in particular the low number of human studies available and the requirement for further in vivo studies with larger sample sizes to support existing data. This, however, further emphasises our rationale of performing a meta-analytical approach.

It was also not our intention to provide guidance for treatment of chronic wounds, for that we would like to refer to the European Society of Clinical Microbiology and Infectious Diseases (ESCMID) guideline for the diagnosis and treatment of biofilm infections. ${ }^{33}$

Another limitation or difficulty with analysing the presence of biofilm in chronic wounds has centred around 'what we define as a biofilm'. Often biofilm are defined based on in vitro observations, and these describe biofilm as bacteria attached to surfaces within a self-produced extracellular matrix and tolerant to antimicrobials. In addition, biofilm development is often described over three to five stages, initiated by planktonic bacteria attaching to a surface, maturation of the biofilm and, lastly, dispersal of bacteria from the biofilm. ${ }^{46}$

However, in vitro observations based on flow cell models using glass surfaces and fresh oxygenated culture media continuously flowing over the bacterium, differ greatly from the conditions within chronic wound infections. ${ }^{34}$ Here, the bacteria are not exposed to a continuous flow of fresh media and are not attached to a glass surface (or to a surface at all). ${ }^{10,39}$ In vivo chronic wound biofilm are also often encapsulated in a matrix, which includes host material, making dispersal problematic.

Therefore, using in vitro observations to define, diagnose and treat biofilm in chronic infections may provide a misguided impression. ${ }^{47}$ There are, however, 
commonalities between in vitro and in vivo evidence that can help in providing a definition of a biofilm. These include: aggregation of bacteria, some sort of matrix that is not restricted to self-produced as it can also be of host origin, and extreme tolerance and protection against most antimicrobial agents and the host defence.

We suggest following this simplified definition in order to define biofilm in chronic infections: an aggregate of bacteria tolerant to treatment and the host defence.

\section{Conclusion}

When combining the results of our systematic review and meta-analysis with the available in vitro models and in animal studies it seems highly likely that biofilms are ubiquitous in non-healing human chronic wounds. It is important therefore, that clinicians appreciate the distinct differences of biofilm phenotypes to their planktonic counterparts, in particular the challenges in eradicating and removing biofilm from a wound. A paradigm shift to a biofilm-based wound care approach should be adopted.
References

1 Hall-Stoodley, L., Stoodley, P. Biofilm formation and dispersal and the transmission of human pathogens. Trends Microbiol 2005; 13: 1,7-10. 2 Ojha, A.K., Baughn, A.D., Sambandan, D. et al. Growth of Mycobacterium tuberculosis biofilms containing free mycolic acids and harbouring drug-tolerant bacteria. Mol Microbiol 2008; 69: 1,164-174. 3 Marsh, P.D., Bradshaw, D.J. Dental plaque as a biofilm. J Ind Microbiol 1995; 15: 3, 169-175. 4 Lam, J., Chan, R., Lam, K., Costerton, J.W. Production of mucoid microcolonies by Pseudornonas aeruginosa within infected lungs in cystic fibrosis. Infect Immun 1980; 28: 2,546-556.

5 Costerton, J.W. Cystic fibrosis pathogenesis and the role of biofilms in persistent infection. Trends Microbiol 2001; 9: 2, $50-52$.

6 Bjarnsholt, T., Jensen P.O., Fiandaca M.J. et al. Pseudornonas aeruginosa biofilms in the respiratory tract of cystic fibrosis patients. Pediatr Pulmonol 2009; 44: 6, 547-558.

7 Donlan, R.M. Biofilm formation: a clinically relevant microbiological process. Clin Infect Dis 2001; 33: 8 , 1387-1392. 8 Hall-Stoodley, L., Hu, F.Z., Gieseke, A et al. Direct detection of becterial biofilms on the middle-ear mucosa of children with chronic otitis media JAMA 2006; 296: 2, 202-211.

9 Boase, S., Foreman, A. Cleland, $E$ et al. The microbiome of chronic thinosinusitis: culture, molecular diagnostics and biofilm detection. BMC Infect Dis 2013; 13: $1,210$.

10 James, G.A., Swogger, E., Wolcott, R. et al. Biofilms in chronic wounds. Wound Repair Regen 2008; 16: 1, 37-44. 11 Bjarnsholt, T., KirketerpMoller, K., Jensen, P.Ø. et al. Why chronic wounds will not heal: a novel hypothesis. Wound Repair Regen 2008; 16: 1, 2-10. 12 U.S Army Medical Department. M.R.a.M.C. Combat Casualty Care Research Program (CCCRP) (2016) http//tinyurl.com/ h6sx8fh (accessed 7 November 2016).

13 Dowd, S.E., Sun, Y., Secor, PR. et al. Survey of bacteria diversity in chronic wounds using Pyrosequencing, DGGE and full ribosome shotgun sequencing. BMC Microbiol 2008; 8: 1, 43.

14 Percival, S.L., MoCarty, S.M., Lipsky, B. Biofilms and wounds: an overview of the evidence. Adv Wound Care $2015 ; 4: 7,373-381$.

15 Wolcott, R.D., Rhoads, D.D. Dowd, S.E. Biofilms and chronic wound inflammation. J Wound Care 2008; 17: 8, 333-341. 16 James, G,A., Ge Zhao. A. Usui, M. et al., Microsensor and transcriptomic signatures of oxygen depletion in biofilms associated with chronic wounds. Wound Repair Regen 2016; 24: 2, 373-383. 17 Wolcott, R.D., Rumbaugh, K.P., James, G. et al Biofilm maturity studies indicate sharp debridement opens a time-dependent therapeutic window J Wound Care 201019 : $8,320-328$

18 Stewart, P.S., Costerton, J.W. Antibiotic resistance of bacteria in biofilms. Lancet 2001; 358: 9276, 135-138. 19 Leid, J.G., Willson, C.J., Shirtliff, M.E. et al. The exopolysaccharide alginate protects Pseudomonas aeruginosa biofilm bacteria from IFN- $\gamma$-mediated macrophage killing. J Immunol 2005; 175: 11, 7512-7518.

20 Gilbert, P., Maira-Litran, T., McBain, A.J. et al. The physiology and collective recalcitrance of microbial biofilm communities.Adv Microb Physiol. 2002; 46: 202-256. 21 Wolcott, R.D., Gontcharova, V., Sun, Y., Dowd, S.E Evaluation of the bacteria diversity among and within individual venous leg ulcers using bacterial tag-encoded FLX and Titanium amplicon pyrosequencing and metagenomic approaches. BM Microbiol 2009; 9: 1, 226-226. 22 Smith, D.M., Snow, D.E. Rees, E. et al. Evaluation of the bacterial diversity of Pressure ulcers using bTEFAP pyrosequencing. BMC Med Genomics 2010; $3: 1,41$. 23 Ganesh, K., Sinha, M. Mathew-Steiner, S.S. et al. Chronic wound biofilm model. Adv Wound Care 2015; 4: 7, $382-388$.

24 Seth, A.K., Geringer, M.R. Hong, S.J. et al. In vivo modeling of biofilm-infected wounds: A review. J Surg Res 2012; 178: 1, 330-338.

25 Kalan, L, Loesche, M., Hodkinson, B.P. et al.

Redefining the Chronic-Wound Microbiome: Fungal

Communities Are Prevalent, Dynamic, and Associated with Delayed Healing.MBio 2016; 7 :

5, pii: e01058-16.

26 Wolcott,R., Sanford, N., Gabrilska, R. et al. Microbiota is a primary cause of

pathogenesis of chronic wound. J Wound Care 2016; 25: Sup10, $\mathrm{S} 33-\mathrm{S} 43$.

27 Davis, S.C., Ricotti, C. Cazzaniga, A. et al. Microscopic and physiologic evidence for biofilm-associated wound colonization in vivo. Wound Repair Regen 2008; 16: 1 , 23-29.

28 Leung, K.P., DArpa, P., Seth, A.K. et al. Dermal wound transcriptomic responses to Infection with Pseudornonas aeruginosa versus Klebsiell pneumoniae in a rabbit ear wound model. BMC Clin Pathol 2014; 14: 1, 20 29 Rumbaugh, K.P., Colmer, J.A., Griswold, J.A., Hamood, AN. The effects of infection of thermal injury by Pseudomonas aeruginosa PAO1 on the murine cytokine response. Cytokine 2001; 16: 4, 160-168. 30 Roy, S., Elgharably, H.
Sinha, M. et al. Mixed-species biofilm compromises wound healing by disrupting epidermal barrier function. J Pathol 2014; 233: 4, 331-343.

31 Percival, S.L., Hill, K.E., Williams, D.W. et al. A review of the scientific evidence for biofilms in wounds. Wound Repair Regen 2012; 20: 5 , 647-657.

32 Liberati, A, Altman, D.G., Tetzlaff, J. et al. The PRISMA statement for reporting systematic reviews and meta-analyses of studies that evaluate healthcare

interventions: explanation and elaboration. BMJ 2009; 339: 1, b2700.

33 Heiby, N., Bjarnsholt, T., Moser, C. et al. ESCMID guideline for the diagnosis and treatment of biofilm infections 2014. Clin Microbiol Infect 2015 21 Suppl 1: S1-S25.

34 Bjarnsholt, T., Alhede, M., Alhede, M. et al. The in vivo biofilm. Trends Microbiol 2013; 21: $9,466-474$

35 Bown, M.J., Sutton, A.J. Quality control in systematic reviews and meta-analyses. Eur $J$ Vasc Endovasc Surg 2010; 40 : 5, 669-677.

36 Martinez-Velasco, M.

Toussaint-Caire, S., HernándezCastro, R., Contreras-Ruizz, J. Biofilm identification and quantification utilizing simple stains and spectrophotometry in chronic wound biopsy samples. Wound Repair Regen 2014; 22 : 2, A53.

37 Honorato-Sampaio, K., Guedes, A.C., Lima, V.L., Borges, E.L. Bacterial biofilm in chronic venous ulcer. Braz Infect Dis 2014; 18: 3, 350-351. 38 Han, A., Zenilman, J.M.,

Melendez, J.H. et al. The importance of a multifaceted approach to characterizing the microbial flora of chronic wounds. Wound Repair Regen 2011; 19: 5, 532-541.

39 Kirketerp-Moller, K., Jensen, P.Ø., Fazli, M. et al., Distribution, organization, and ecology of bacteria in chronic. J Clin

Microbiol 2008; 46: 8 , 2717-2722.

40 Oates, A., Bowling. F.L., Boulton, A.J. et al. The Visualization of Biofilms in Chronic Diabetic Foot Wounds Using Routine Diagnostic Microscopy Methods. J

Diabetes Res 2014; 2014 : 153586.

41 Trostrup, H., Thomsen, K. Christophersen, L.J. et al. Pseudomonas aeruginosa biofilm aggravates skin inflammatory response in BALB/c mice in a novel chronic wound model. Wound Repair Regen 2013; 21: 2, 292-299.

42 Fazli, M., Bjarnsholt, T. Kirketerp-Moller, K. et al. Quantitative analysis of the cellular inflarnmatory response against biofilm bacteria in 